\title{
Stereoscopic TR PIV measurement and POD analysis of flow behind the turbine prototype of model size
}

\author{
Darina Jasikova ${ }^{1,}$, , Michal Kotek, and Vaclav Kopecky \\ ${ }^{1}$ Technical University of Liberec, Studentska 2, 46117 Liberec, Czech Republic
}

\begin{abstract}
When measuring turbine prototype flow, a number of modes with different flow rates and cavitating vortex rope settings have been measured. The turbulent flow in the draft tube of diameter $240 \mathrm{~mm}$ reaches the Reynolds number of $10^{5}$. The vortex and highly swirling flow is developed in the draft tube. The usage of $\mathrm{kHz}$-order frame rates was necessary to resolve the velocity field. The investigated area in the mixing vessel was examined by the stereoscopic time-resolved PIV technique. Further mode evaluation allows comparison of cross-sectional velocities in the vector map. The Proper orthogonal decomposition analysis was applied on $3 \mathrm{D}$ vector maps, those including $\mathrm{u}, \mathrm{v}, \mathrm{w}$ velocity compounds. In the selected regimes - operating points with volume flow rate $97 \mathrm{l} / \mathrm{sec}$ and $114 \mathrm{l} / \mathrm{sec}$, the greatest risk of transition to cavitation mode was identified, so they were studied in detail.
\end{abstract}

\section{Introduction}

The development of energy storage re-sources is a response to weather fluctuations and growth of green power plants (solar and wind power). There raises an urgent need for energy storage and its use depending on the immediate increase. It is also very closely connected with ensuring the safety and stability of the distribution system. The most effective functions are assured by pumped-storage hydroelectric power plants (PVE). The unique feature is their rapid response to the needs of the transmission network and the ability to ensure their re-commissioning in case of blackout. In Europe, most of the PVEs were built more than 20 years ago, i.e. before volatile renewable resource boom, where the requirements for dynamic PVE functions and bandwidth were less demanding than today. The number of PVEs installed in Asia and South America has been so far inadequate. The usage of conventional reverse turbines in off-optimal modes brings an unstable closed flow causing the vibration pulsation of the turbocharger. It forces the limitation of the working scope.

The progress in turbine optimization is in a design of new shape of the hydraulic parts of the reverse turbine, assuming the rotation of the fluid in the draft and suction tube. The design of impeller, draft and suction tube of reverse (pump) turbine with an extended operating band is based on computational modelling (CFD, mathematical optimization).

* Corresponding author: darina.jasikova@tul.cz 
Validation of models can be performed by advanced experimental techniques (timeresolved PIV, tomography, high speed visualization).

The intention in progression is to suppress pulsations, which are the result of the cavity of the swirling head. The novelty of the reverse turbine design is in optimization of the plant as a whole unit (impeller plus suction unit) using computational modelling with help of advanced experimental techniques. The solution is based on a thorough theoretical analysis on stability and flow dynamics. The verification of the prototype runs in testing room on a reverse turbine prototype. This prototype meets requirements and demands of dynamic control function, thereby increasing the safety and resilience of the distribution network.

The validation of the numerical calculations should be done by the method that allows nonintrusive contactless measurement of fluid flow meeting the temporal resolution of an unsteady flow. The turbulent flow in the draft tube of diameter $240 \mathrm{~mm}$ reaches the Reynolds number of $10^{5}$. The vortex and highly swirling flow is developed in the draft tube. The usage of kiloHertz-order frame rates was necessary to resolve the velocity field. Another condition influencing the selection of the appropriate measuring methodology was the $3 \mathrm{D}$ character of flow. The flow behind the turbine is anisotropic. The velocity component in the mainstream direction greater then swirl flow. The time-resolved PIV (TR PIV) can provide access to spatial - temporally varying flow fields at sampling rates of $\mathrm{kHz}$. The mainstream time-mean velocity was calculated from volume flowrate as $W_{0}=2 \mathrm{~m} / \mathrm{s}$ and the velocities in swirl flow ware expected as $U_{0}$ and $V_{0} 0.3 \mathrm{~m} / \mathrm{s}$. Measurement in two planes was impossible for spatial reasons and optical approach to the prototype. Therefore, a one-off measurement was provided to get information on all three velocity flow components. Confirming the expectations was to use stereo PIV system setup know from conventional PIV system. Here, we set the stereo TR PIV (3D TR PIV) system on Nd:YLF laser Litron running on maximal flashing frame $1 \mathrm{kHz}$ in double frame mode and two high speed cameras set in 3D geometrical arrangement using Scheimpflug mounts. The results of fluid flow presented in this paper are complemented by proper orthogonal decomposition calculated for three velocity components [1-7].

\section{Experimental Method}

The selection of the measuring methodology and experiment conditions on the turbine prototype were determined by the high $\operatorname{Re}$ number $\left(\operatorname{Re}>10^{5}\right)$ and the anisotropic character of the fluid flow in the draft tube. The diameter of draft tube at the measuring plane was $240 \mathrm{~mm}$. Average expected water flow through the cross section was $100 \mathrm{l} / \mathrm{sec}$.

The time - resolved PIV extended with two high speed cameras to obtain stereo records of fluid flow was suitable solution for monitoring of three velocity component using two optical accesses. The experimental measurement was run in the test hall on the turbine prototype. Together with the design of the geometric arrangement of the cameras and the laser, it was necessary to design a suitable optical approach and to supplement the tube with the optic extensions. According to requirements of optical measuring methods, the prototype of suction and draft tube was manufactured with Plexiglas to ensure optical access also for purpose of visualizations. It was mainly the reduction of the distortion caused by the transition of light through various environments (water, Plexiglas, air) and curved walls of draft tube. 

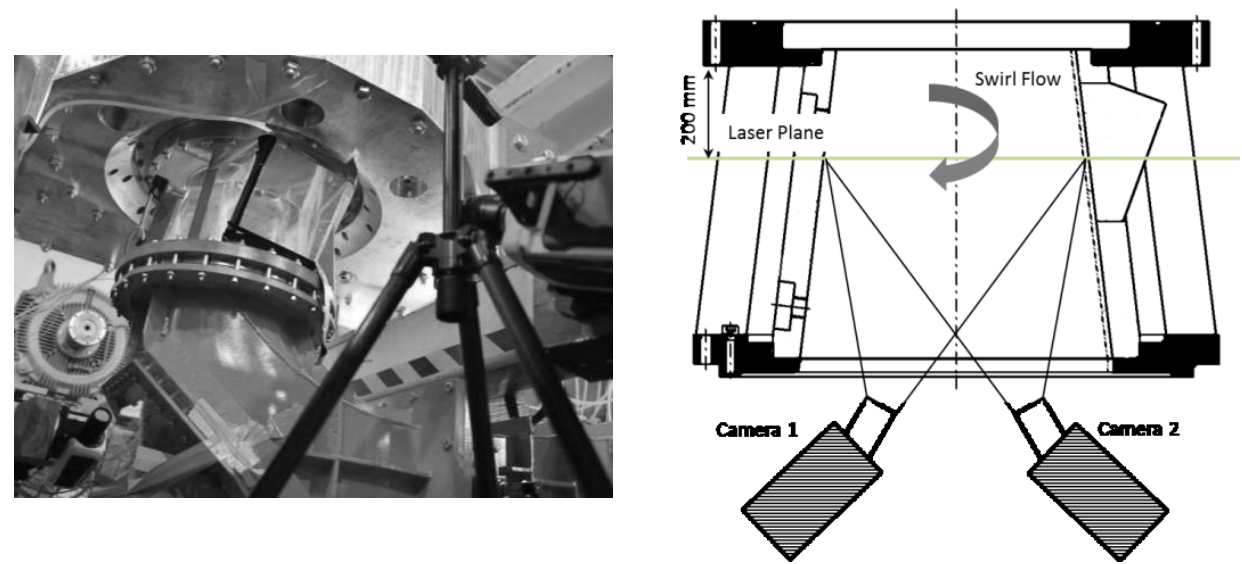

Fig. 1. Photo of TR PIV system layout - two cameras in the bottom view. There is seen a window for equalizing different refractive indices of the environment and curvature of draft tube. Scheme of draft tube geometry behind the impeller and location of the laser plane and stereoscopic TR PIV camera setup.

\subsection{Measurement Technique Setup}

The investigated area in the mixing vessel was examined by the time-resolved PIV technique. The stereoscopic TR PIV system was built on two high speed Speedsense cameras, complete with a sync module and a Litron Ld:Y300 pulse laser operation on the frequency $1 \mathrm{kHz}$. This kind of double cavity laser emits pulses of energy reaching $15 \mathrm{~mJ}$ in each pulse on wavelength $527 \mathrm{~nm}$. The laser beam was extended into the vertical plane with cylindrical optics to reach the parameter of the planar laser sheet of thickness $1 \mathrm{~mm}$ and spread into the $300 \mathrm{~mm}$ width. The investigated area's diameter was $240 \mathrm{~mm}$.

The two high speed cameras SpeedSense working on frequency $1 \mathrm{kHz}$ with resolution of $(1280 \times 800) \mathrm{px}$ in double frame mode were equipped with low-passing filters to eliminated the backward flashes from the laser sheet that arises on the blades surfaces. The wavelength of the optical filter corresponds with the emitted light of the particles. The set of lens system Nikkon $\mathrm{f} / 2.860 \mathrm{~mm}$ was also used. The cameras were fitted into optical amounts to reach Scheimpflug condition. Conventional particle image velocimetry uses a single camera oriented orthogonally to the illuminated plane. Stereoscopic simultaneous views from two different off-axis directions provide sufficient information to extract the out-of-plane and in-plane velocity components. We used the angulardisplacement method in our experimental camera setup. That means that two cameras axes are not parallel, but rotated inward such that they intersect at the midpoint of the domain to be recorded. The object plane is not parallel to the lens plane Therefore it becomes difficult to obtain particle images that are well focused across the image plane. Most simple solution is to increase the depth of field of the recording optics. The depth of field $d o f=$ $4\left(1+M^{-1}\right)^{2} f^{2} \lambda$, where $M$ is the camera magnification, $f$ is the f-number, and $\lambda$ is the wavelength of the illuminating laser. A large depth of field can be obtained of increasing the f-number, implying that a smaller fraction of the light scattered by the particle will reach the camera. The second method to obtain images in focus over the entire image plane is to enforce the Scheimpflug condition. That requires the object plane, the lens plane, and the image plane are collinear. We used both methods with regard to insufficient saturation in our experiment.

The exposition time for both cameras operated on 330 usec. The increase of exposure time increases the particle density and illumination. 
The laser and camera system was synchronized via timer box and controlled from the DantecStudio software. The expectation of the maximum frequency of the vortex structures was in tens of Herz. According to Nyquist criterion of sampling frequency it was set $500 \mathrm{~Hz}$. The dataset of 5000 images was captured. The raw images were also processed in this software.

The analyses of captured images run in three main categories. There were run pre processing analysis, i.e image masking, image algorithm for blur and light reflection reduction. There was run the dewarping algorithm with the calibration function. The preprocessed images were analysed using standard cross-correlation method with interrogation area size $(32 \times 32) \mathrm{px}$ and overlap $50 \%$. The raw vector maps were validated with peak and range validation methods to obtain correct dataset. For the purpose of overview of the complex flow behaviour the statistical evaluation and the turbulence index was calculated. The dynamically changing velocity field was analysed by the Proper Orthogonal Decomposition (POD) for identifying the energy fractions and partial vortex structures in the fluid flow.

\subsection{Calibration}

The stereoscopic TR PIV requires spatial calibration with a specially designed calibration target. The use of a calibration target with an accurate dot matrix also enables subsequent post processing of captured images and dewarping of local distortions.

Standard calibration of the stereo PIV system is performed by a traversing calibration target. The movement of the target in a closed floating waterway could not be realized, therefore a multi-level target method was chosen.

The calibration target was designed as a spatial matrix with regular point alternation in two planes. When designing a target, it was important to keep the square structure of both planes, which later defined the axial orientation. The designed round calibration target was manufactured using 3D printing method. This method enables precise two colour printing with high resolution. The appropriately designed target must have the following parameters: number of pixel points in the image $10 \times 10$, the ratio of the size of the main points to the image width of 1:50, the ratio of the main points to the central $1: 1.5$, and the ratio of the main point size and 1: 1 layer spacing. The target was designed as white dots on a matt black background. The main dot size was $7 \mathrm{~mm}$ and the regular dot was $5 \mathrm{~mm}$ in diameter. The distance between dots was $20 \mathrm{~mm}$ and the plane distance was $4 \mathrm{~mm}$.
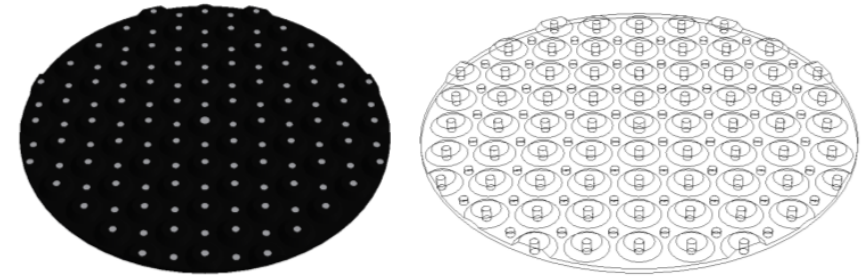

Fig. 2. Calibration target with a diameter of $240 \mathrm{~mm}$ for measurement in the experimental track.

\subsection{Seeding}

Solution for seeding was first supposed to use a natural flow of saturation. This was especially content of impurities and bubbles that have proven themselves in the past. The advantage of natural saturation was more considerate to the device. There was a legitimate concern about blade damage and the deposition of particles in the system. There was a problem with the seeding of the fluid flow due to the spatial layout of two cameras to the laser plane from experimental point of view. Each camera records the 
evaluated area at a different angle to the scattering angle on the particle. The problem was the diffusion of light at different angles. The bubbles show a backscatter of laser light. Solid particles, on the other hand, exhibit forward scattering. However, the natural impurity content was not sufficiently proportional for both cameras to record particles of sufficient intensity, notwithstanding the fact that two non-comparable seeding units (density, viscosity, reflection index) were captured. The stereoscopic TR PIV method is particularly sensitive to the quality of seeding, especially when it comes to high-speed recording with short exposure times and limited light power. These arguments outweighed concerns about the damage to the system, which was not proven later. The volume of $50 \mathrm{~m}^{3}$ (whole volume in the turbine, pipe system and reservoir) of water was to be seeded with $50 \mu \mathrm{m}$ PA particles.

\section{Camera 1 - forward scattering Camera 2 - backward scattering}

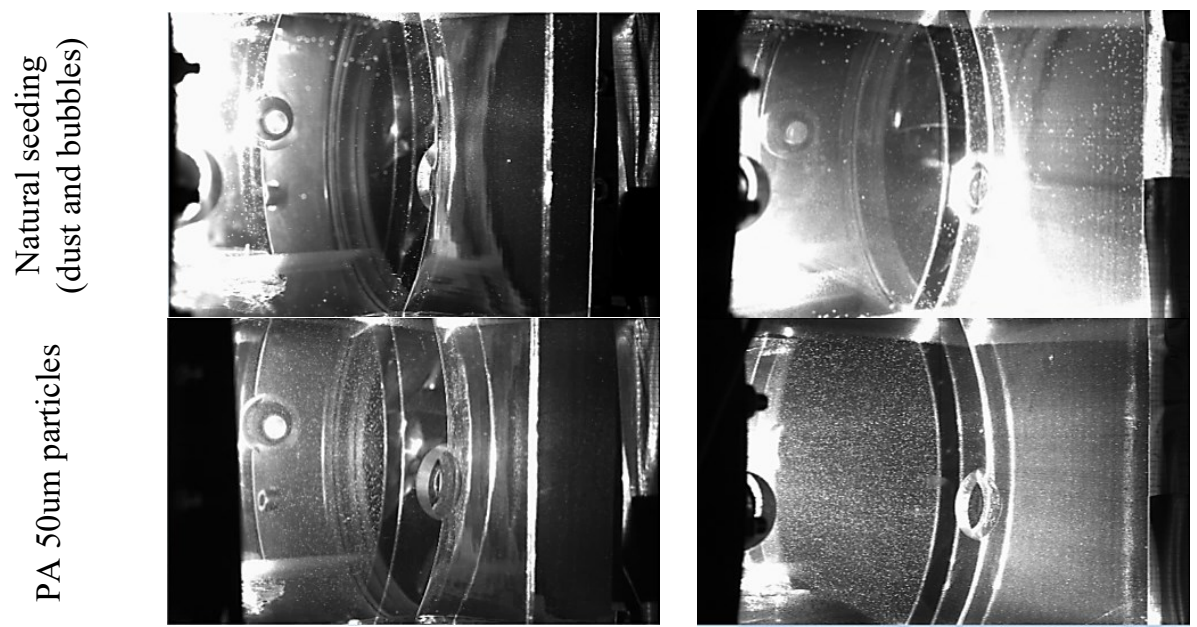

Fig. 3. Examples of seeding using presence of natural dust, impurities and bubbles, and addition of PA 50um particles.

\section{Results and Discussion}

When measuring turbine prototype flow, a number of modes with different flow rates and cavitating cavity settings have been measured. This article lists results from two modes that are most prominent. There is a risk of an early transition to the cavitating character of the fluid flow (due to under pressure) for both operating modes. The impeller optimization design was based on the suppression of the cavitating whirl. In the selected regimes operating points marked as 293 and 298, the greatest risk of transition was identified, so they were studied in detail.

Mode 293 was running at fluid flow $97 \mathrm{l} / \mathrm{sec}$ with a slight overpressure of $20 \mathrm{kPa}$. In this mode, the cavitating rope did not go into the gaseous phase, so it was possible to perform PIV measurements. By lowering the pressure of $20 \mathrm{kPa}$ under atmospheric pressure, the core of the cavitating whirl changed the phase to the gas occupies a large part of the evaluated area. Condition for PIV measurements are harder to reach due the reflections and lack of seeding inside the cavitating rope. Mode 298 is characterized by a flow of $114 \mathrm{l} / \mathrm{s}$ at atmospheric pressure. This mode was stable under current operating conditions.

The stereoscopic model used to reconstruct three component vector maps was loaded with a number of limits. First and foremost, due to the complexity of the scene and the 
views to the measured area, it was not possible to cover the entire cross-section of both cameras. In places where the view of a single camera is hidden, it is not possible to reconstruct the data properly. This leads to misinterpreted results, especially in some extreme areas. However, most of the cross-section is interpreted correctly and it is certainly possible to have the overall flow.

The basic evaluation of fluid flow behind turbine is time-averaged statistics (Figure 4). We captured in the following vector maps for following measurement points 293, and 298. The basic physical quantities of the operation were recorded at all of these measurement points.

$\mathrm{a}$
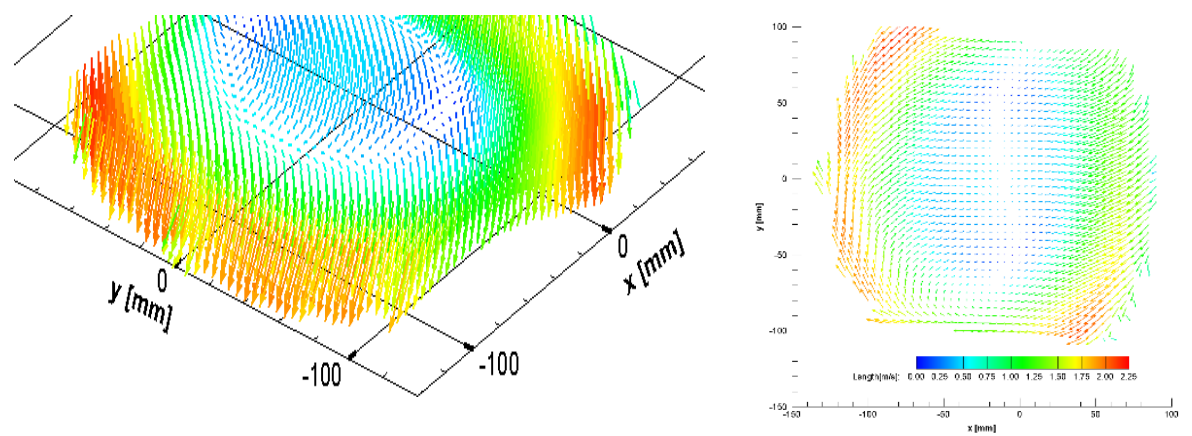

b
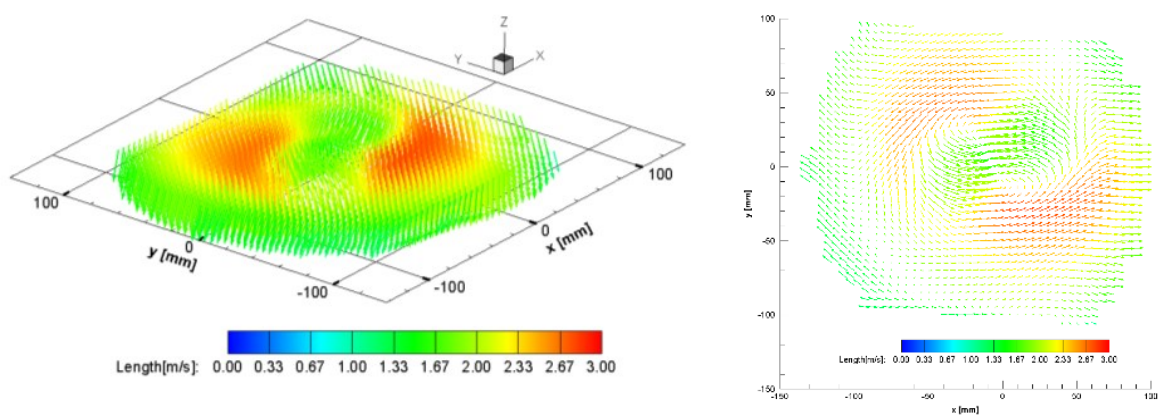

Fig. 4. Time-averaged statistics of fluid flow behind the turbine prototype processed as $3 \mathrm{D}$ and $2 \mathrm{D}$ vector maps for regime a) 293, and b) 298.

From the basic view of the vector maps, there is a difference in the mode at the measurement point 298. While in the other modes exhibits the dominant stream of water is in the peripheral regions, at the point 298 mode, the main stream with the vortex structure moves closer to the centre of the cross-section. The maximum velocity are higher in this mode, $w_{\max }=3 \mathrm{~m} / \mathrm{s}$. An interesting comparison of paragraphs 298 and 293 shows another difference in the characteristics of the modes.

There is a difference between rotations of the main current behind the impeller from this interpretation of the results (Figure 4). While in the modes at points 293 rotates the main current according to the direction of the impeller, in this case in the clockwise direction, the mode at point 298 rotates in the opposite direction of the impeller. This behaviour of the vortex structures was subsequently confirmed by high-speed visualizations in the presence of the gaseous component.

Further mode evaluation allows comparison of cross-sectional velocities in the vector map. 
The Proper orthogonal decomposition analysis was applied on 3D vector maps, those including $u, v, w$ velocity compounds. It is a mathematical method that gains and clarifies the flow structures based on extracting a basis for modal decomposition from an ensemble of signals. [8] We can reconstruct flow field and demonstrate the influence of different flow structures by the selection of main dominant POD modes. The major POD modes that effects the dominant flow is seen in Figure 5. The POD Snapshot is based on autocorrelation function and depends on the number of instantaneous acquired samples acquired. We can see in figure 5 two dominant Modes that are significant for flow regime 293. For comparison to this we chose the first dominant mode even for the regime 298.
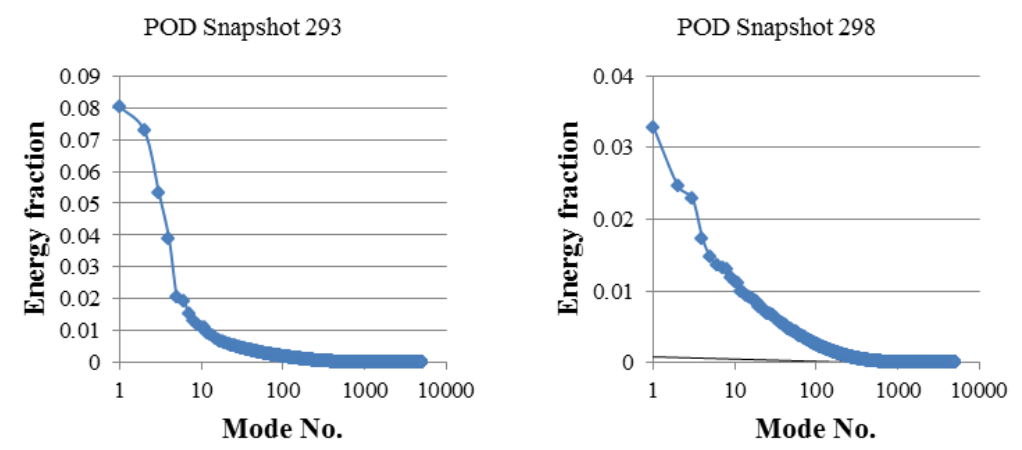

Fig. 5. POD Snapshots for regime 293, and 298 according to energy fractions.

The general aim of POD is to find the optimal representation of measured field realizations. The eigen functions are sometimes referred to coherent structures. The structures are highly correlated in an average sense with the flow field. The POD analysis was performed on 5000 sequential 3D vector maps. The POD projection of Mode 1 and 2 for both regimes is seen in Figure 6 and Figure 7. These two dominant modes affect the resulting flow structure. The presentation of all three components of velocity, i.e. $u, v$ in plane and $w$ as vertical velocity component, is also evident in anisotropy and orientation of the main and swirl flow.

The interpretation of $3 \mathrm{D}$ vector velocity fields is difficult, especially if they are strongly anisotropic. In this experiment, the component of the vertical velocity $w$ was predominantly in the mainstream direction, so it follows the main swirl flow. We focused on viewing this folder, when interpreting data.

In regime 293, the centrifugal flow orientation is predominant, resulting in a structure with a maximum velocity in the laser plane ( $u, v$ velocity components) - horizontal, near the walls. The rotational frequency of these structures is $7 \mathrm{~Hz}$. The swirl structure and centred local velocity decrease is dominant in both POD modes. The return fluid flows in the centre time-averaged velocity statistics is created by averaging these vortex structures, which rotate with the main stream. The increase of velocity near the walls is well visible on the projection of 3D images in figure 6 .

In regime 298 in POD mode 1, and in 2 prevail the vortex structures in the centre of swirl structure. Complex, irregularly structured vortex are projected into time-averaged vector statistics as two opposite vortex structures moving in counter clockwise direction of rotation. In averaged statistics, these partial vortex structures cause a uniform velocity profile as it is seen in figure 8. The occurrence of both POD modes is time-based as it is seen in figure 7 . This local closure predicts the incidence of cavitation rope if pressure drop. 

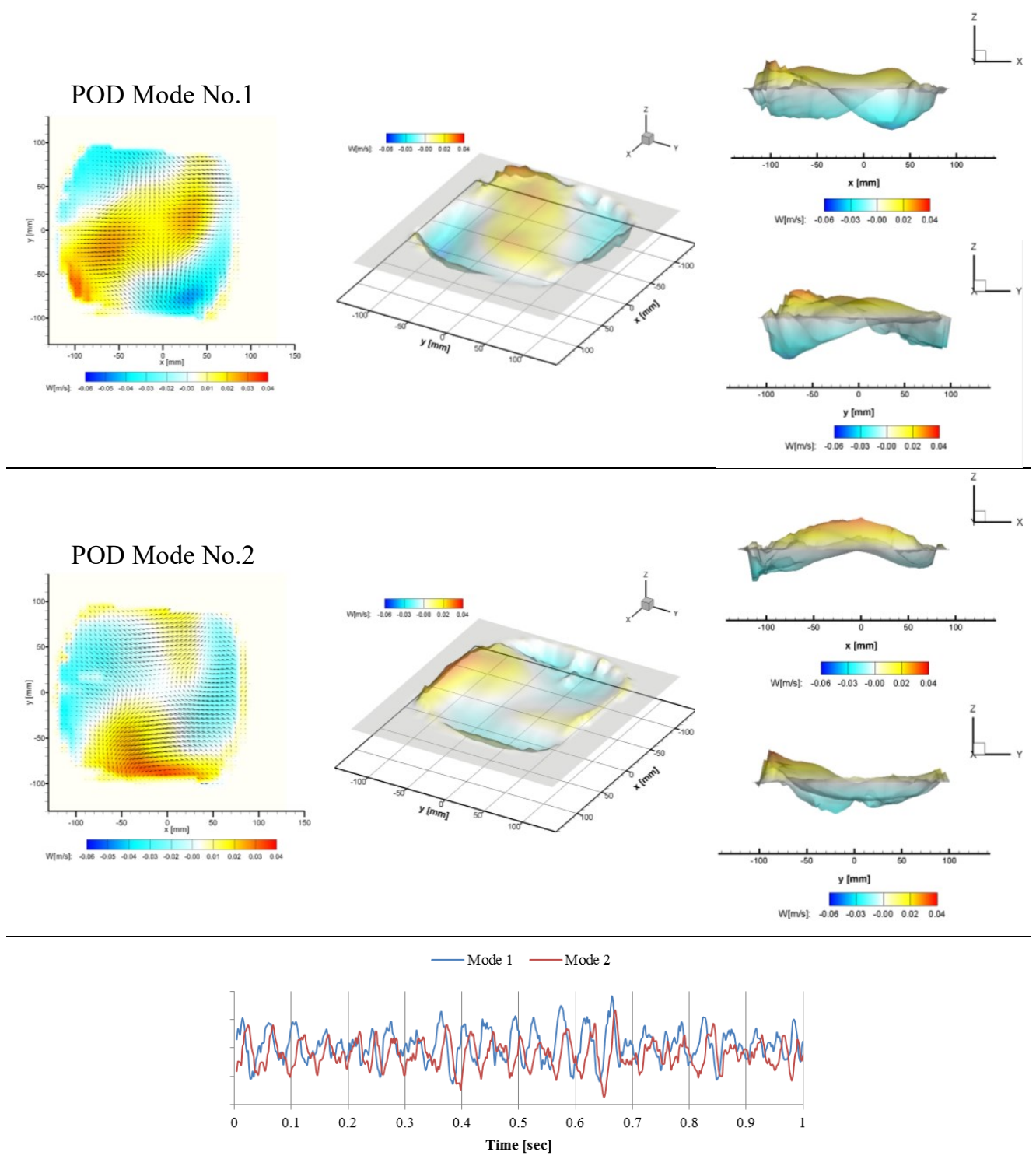

Fig. 6. POD $1^{\text {st }}$, and $2^{\text {nd }}$ mode of flow structures in regime 293 and time history of POD coefficient for both modes.

There is seen significant vortex breakdown. This breakdown is characterised by decrease of axial velocity along the axis, leading to internal stagnation point or even backflow [7].

Two modes differing in flow, achieve significant different flow characteristics, which are also reflected in the speed profile evaluation that was taken across the transverse region passing through the centre of the diffuser. According to the velocity profiles for vertical velocity component $w$ in Figure 8 can be watched the dominant trend of the flow reaches the maximum near the wall of the diffuser. Towards the centre of the diffusers, the velocity of the velocity decreases and there is a backward flow and significant closure in the horizontal plane. 
Against this mode 298 towards the centre of the diffuser, the speed increases, including the rotary component, see vector map above. The small drop is due to the rage behind the impeller's charge.
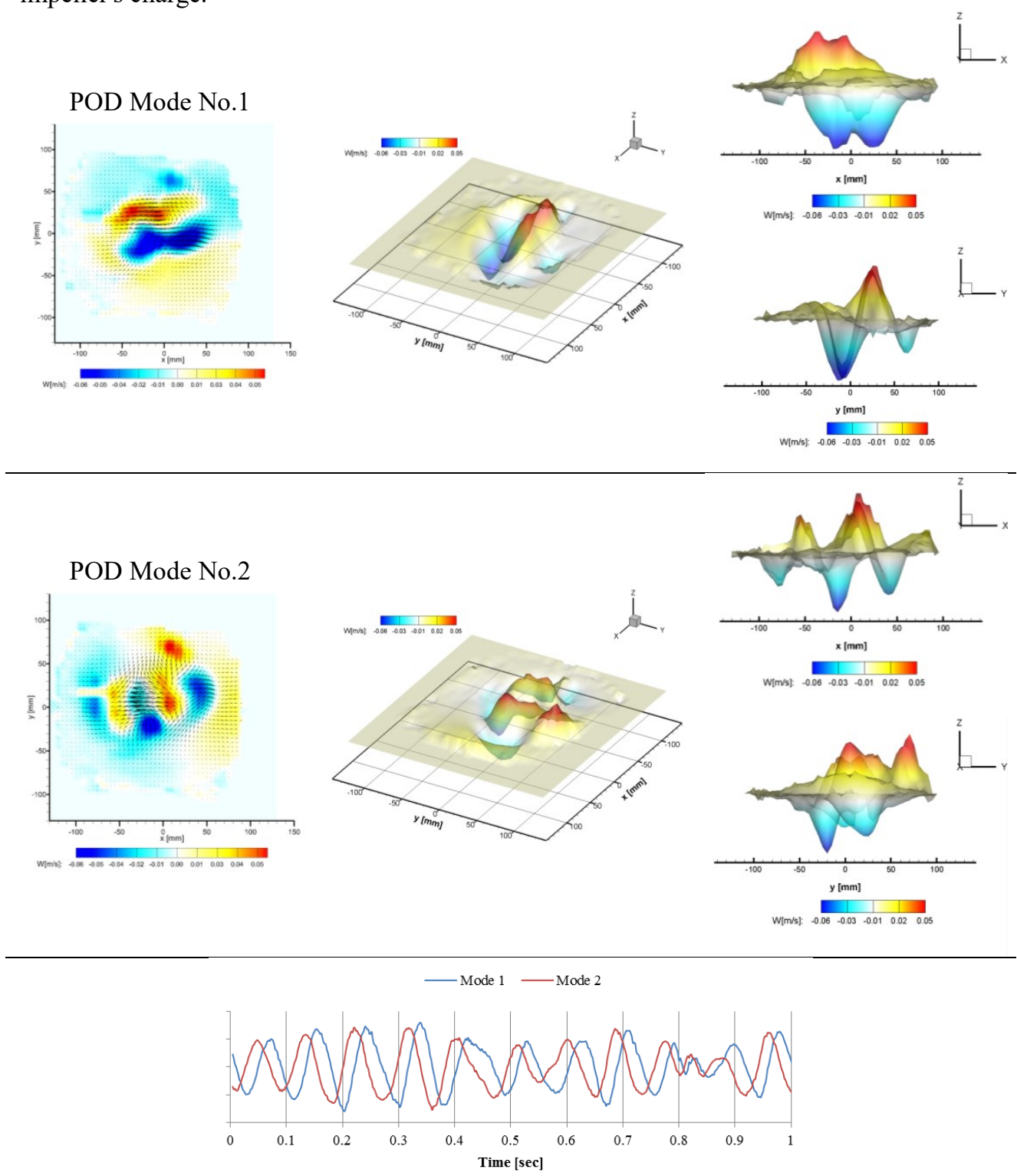

Fig. 7. POD $1^{\text {st }}$, and $2^{\text {nd }}$ mode of flow structures in regime 298 and time history of POD coefficient for both modes. 

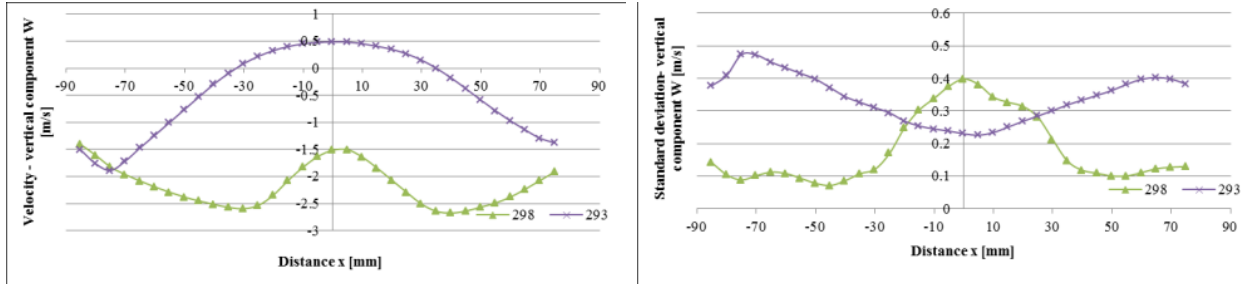

Fig. 8. Profile plots of velocity component $w$ and standard deviation Std $w$ taken across the measured area under the impeller.

\section{Conclusion}

The measurement in draft tube below the impeller was a technically demanding. In addition, it has to be solved with compensating optical elements. The distortion near the curved walls was too large to be able to measure the speed field in these areas. The evaluation of field speeds near the wall provides the most interesting results, especially in the area behind the rotating impeller, when the main current is closed.

Using optimized PIV stereo methods and high speed recordings, it was possible to monitor and evaluate this complex task as well. By application of the POD analysis, dominant vortex structures were detected, which were evaluated in the form of 3D graphs. The distinction between the two modes is reflected not only in the evaluation of the velocity profiles across the monitored area, but also in the resulting frequency analysis, where the dominant frequencies of the vortex structures can be detected. This measurement responds to the CFD simulation already conducted to optimize the turbine.

The results of this project LO1201 were obtained through the financial support of the Ministry of Education, Youth and Sports in the framework of the targeted support of the "National Programme for Sustainability I".

\section{References}

1. A. Upatnieks, K. Laberteaux, S. L. Ceccion, Experiments in Fluids 32 (2002)

2. S. Wang, Y. Chen, Y Z. Li, J Vis 21 (2018)

3. D. F. Kurtulus, F. Scarano, L. David, Exp Fluids 42 (2007)

4. L.L. Shi, Y.Z. Liu, J.J. Wan, Journal of Mechanical Science and Technology 24 (2010)

5. J. Basley, L.R. Patur, F. Lusseyran, Exp Fluids 50 (2011)

6. A. Villegas, F.J. Diez, Exp Fluids 55 (2014)

7. P. Rudolf, D. Štefan, IOP Conf. Series: Earth and Environmental Science 22 (2014)

8. L. Sirovich, Quarterly of applied mathematics 3 (1987) 